\title{
Post-traumatic rehabilitation of dogs after limb fractures with correction of vitamin and mineral metabolism
}

\author{
N.V. Shamsutdinova, G.G. Shalamova*, E.V. Smelkova, R.M. Papaev, and N.R. Kasanova \\ Kazan State Academy of Veterinary Medicine named after N E. Bauman, city of Kazan, Russian \\ Federation, guze1471979@mail.ru
}

\begin{abstract}
The parameters of the level of calcium, phosphorus and vitamin $\mathrm{D}_{3}$ in the blood of injured dogs aged 6 to 8 months were studied. In all animals with limb fractures, the levels of $\mathrm{Ca}, \mathrm{P}$, and vitamin $\mathrm{D}_{3}$ are reduced, which led to bone fragility. We used a conservative method of treatment with the application of a plaster cast and medical treatment aimed at relieving inflammation, pain and formation of a callus. After removing the plaster cast, all the animals had joint stiffness, soreness, and partial muscle hypotrophy. A rehabilitation program was developed, which included: physical exercises, massage. This led to a rapid recovery of limb muscles and an increase in the volume of movements.
\end{abstract}

\section{Introduction}

Injuries among small domestic animals are quite common, with bone fractures accounting for the largest proportion.

Each bone is formed from two types of tissues: cortical-compact, located on the periphery, and spongy. The ratio of these bone tissue components is not constant and depends on the shape, type of bone, as well as on the age of the animal and the state of its health. Bone tissue has metabolic activity, biological plasticity, regeneration potential, ability to morphologically rearrange, functionally transform and recover from damage without a connective tissue scar with full-fledged bone tissue. It is constantly undergoing restructuring - the resorption of some and the formation of other, new structures of ostein [1].

In case of bone fracture in animal, there is a strong pain reaction in the first days in the form of aggression, restriction of movement, and solitude [2].

Treatment of fractures is carried out by conservative and operative methods. A tight bandage, plaster cast can be applied or osteosynthesis can be performed [3, 4]. Immobility of the limb for callus formation and healing of soft tissues is temporarily created.

The formation of bone callus depends on the vitamin-mineral, carbohydrate, and fat metabolism in the body [5].

In the skin of animals, vitamin $\mathrm{D}_{3}$ (cholecalciferol) is formed, which stimulates the absorption of calcium and phosphorus from the intestine, increases their concentration in

\footnotetext{
* Corresponding author: guzel471979@mail.ru
} 
the blood and deposition in bone tissue. With vitamin D deficiency, even with normal intake of $\mathrm{Ca}$ and $\mathrm{P}$ with a diet, bone fragility develops, which leads to the development of rickets, osteoporosis [6].

During the treatment of fractures, even with the formation of a bone callus, partial atrophy of limb muscles occurs, the work of the ligamentous apparatus is disrupted due to prolonged adynamia. There is also a decrease in muscle strength and endurance, an impaired coordination of movements, which can later lead to contractures, tendency to injure another part of the body and repeated injuries. [7].

In clinical medicine, physical rehabilitation is widely used to restore functional abilities. Physical rehabilitation is the use of physical exercises and natural factors for therapeutic and preventive purposes in the complex process of restoring the health, physical condition and working capacity of patients and disabled people. It is an integral part of medical rehabilitation and is used in all its periods and stages. The process is carried out in a complex way under the influence of specially selected physical exercises, massage, water and physiotherapy procedures [8].

Rehabilitation with the help of physical exercises is a set of exercises that help to regain the lost physical movement ability. Exercises depend on the location of the injury. After physical exercises, significant changes occur in the body: the metabolic processes in the muscles improve: due to an increase in oxygen and nutrients supply, blood circulation at the area of injury accelerates; edema decreases, the body's adaptation to various influences improves and optimizes the functional activity of internal organs [9].

Rehabilitation can also be carried out with the use of water treatments, massage, which stimulate the activity of all body systems after injuries. Currently, kinesio taping is being actively implemented. For this purpose, a cotton elastic band (tap) is used, which is attached to the skin, to reduce compression at the area of injury. Physiotherapy is widely used, which includes: magnetic therapy, electrical stimulation, warming up, laser therapy [10].

Massage is one of the most important means of musculoskeletal system diseases treatment. It is prescribed in case of bruises with hemorrhage, sprains of muscles, ligaments and tendons, fractures, dislocations, etc. Massage helps to relieve pain. Thanks to it, edema decreases, hemorrhage resolves, muscles are strengthened, joint and muscle functions are restored, callus forms faster, etc. [10]

All rehabilitation methods are aimed at restoring the function of damaged muscles and organs in humans. Despite the widespread use of rehabilitation in clinical medicine, the problem of rehabilitation in veterinary medicine is not sufficiently studied.

We have studied the state of mineral metabolism in bone fractures in dogs, the method of its correction was determined, and a program of post-traumatic rehabilitation of animals was compiled.

\section{Materials and methods}

Studies were conducted on 5 outbred dogs aged 6-8 months with fractures of the limbs of different localization.

The diagnosis was made on the basis of clinical signs and X-ray examination.

$\mathrm{X}$-ray studies were performed using the DongmumDIG-360 device with a Rayence1717SGC flat-panel X-ray detector.

A conservative method of treatment of limb bones fractures was used, including: application of a plaster cast and medical treatment aimed at relieving inflammation, pain and the formation of a callus.

To study the biochemical composition, blood was taken on an empty stomach in the morning in biochemical tubes at a dose of $1 \mathrm{ml}$ from the medial saphenous vein of the 
lower leg, observing asepsis rules. The blood test was performed three times: on the first day and on the 14th, 30th day of treatment. The study of vitamin $\mathrm{D}_{3}$ presence was carried out on an ABBOTT ArchiteetI $200025-\mathrm{OH}$ analyzer. The study of $\mathrm{Ca}$ and P level in the blood was carried out on a StatFax 3300 biochemical analyzer.

\section{Research results}

Our studies involved 5 dogs aged 6 to 8 months. The conditions of birth and keeping of animals in the early postnatal period are not known, since 3 dogs were purchased from previous owners, and 2 were picked up on the street.

The period of animal body growth occurred in the winter and spring months. The dogs were kept in different conditions - 3 in an apartment, one on a balcony, and one in a crate. All animals were dehelminted and vaccinated. 3 dogs were fed with special Purina, Proplan feeds, and 2 dogs - with home-made food (cottage cheese, fish, porridge, meat).

During the entire period of growth, three dogs were not taken out on the street, they were injured as a result of a fall, and two were found with broken limbs.

During the clinical examination, the temperature, pulse, and respiration of all the animals were within the normal range. Two dogs were very worried, behaved aggressively, 3 dogs had apathy. When moving, all the animals were supported on three limbs, and the damaged one was held in the air. In palpation of the injured limb, the animals reacted aggressively due to the pain response. There was swelling of the muscle tissue and crepitation of bone fragments at the site of fracture. Skin damage was not observed in any animal.

All animals underwent radiographs depending on the location of the fracture, on the basis of which diagnoses were made:

1. Closed diaphyseal fracture of the radius and ulna - 2 dogs.

2. Closed fracture with displacement in the proximal femur -2 dogs.

3. Closed metaphysical fracture of the tibia and fibula and a fracture of the metaphysical part of the femur with displacement - 1 dog.

For the treatment, a conservative method was used with the application of a plaster cast and the use of medications, the action of which is aimed at relieving inflammation, pain and callus formation. The immobilizing bandage was applied to 4 dogs for 4 weeks, and 1 - for 6 weeks.

The following drugs were used for medical treatment:

- as a sedative - xylazine hydrochloride in a dose of $0.1 \mathrm{ml} / \mathrm{kg}$ intramuscularly;

- for local anesthesia - $0.5 \%$ novocaine solution

- to prevent the development of secondary microflora - antibiotic bicillin - 3 at a dose of 10,000 units per $\mathrm{kg}$ of animal weight, once;

- as an anti-inflammatory drug - fluniject at a dose of $0.02 \mathrm{ml} / \mathrm{kg}$, intramuscularly once a day for two days;

To study the mineral metabolism of all the injured animals, blood was taken and a biochemical analysis of the levels of $\mathrm{Ca}, \mathrm{P}$ and vitamin $\mathrm{D}_{3}$ was performed. This is necessary, since calcium and phosphorus ions play a crucial role in the formation of bone tissue structure and the functioning of the musculoskeletal system, which performs protective and supporting functions. The bone deposits significant amounts of calcium and phosphorus in the form of microcrystalline and amorphous hydroxyapatite, which provide the strength and structural characteristics of the bone. The main function of vitamin $\mathrm{D}_{3}$ is to maintain normal concentrations of calcium and phosphate in the blood plasma, which are necessary for adequate bone mineralization and skeletal formation. During the study, it was found that the indicators were reduced: $\mathrm{Ca}-2.0 \mathrm{mmol} / \mathrm{l}, \mathrm{P}-0.79 \mathrm{mmol} / \mathrm{l}$, Vitamin $\mathrm{D}_{3}-95$ 
nmol/1 (normally: Ca - 2.6-3.0 mmol/l; P - 1.2-2.5 mmol/l, vitamin $\mathrm{D}_{3}-100-120 \mathrm{nmol} / \mathrm{l}$ ), which led to bone fragility.

To normalize the level of vitamin $\mathrm{D}_{3}$, calcium and phosphorus, the drug "Vitatrin" was used: for puppies 2-4 drops 1 time a day for 4 weeks with food; "Fitokalcevit for puppies" for puppies weighing up to $8 \mathrm{~kg}, 1$ teaspoon with food 1 time a day daily for 4 weeks. During the treatment, blood was re-taken for analysis on the 14th and 30th days (Table 1, Fig. 1, 2).

Table 1. The content of calcium, phosphorus and vitamin $\mathrm{D}_{3}$ in the blood of dogs

\begin{tabular}{|l|c|c|c|c|}
\hline \multicolumn{1}{|c|}{ Indicators } & norm & Day 1 & Day 14 & Day 30 \\
\hline $\mathrm{Ca}(\mathrm{mmol} / \mathrm{l})$ & $2.6-3.0$ & $2.0 \pm 0.9^{*}$ & $2.2 \pm 0.7^{*}$ & $2.5 \pm 0.5^{*}$ \\
\hline $\mathrm{P}(\mathrm{mmol} / \mathrm{l})$ & $1.2-2.5$ & $0.79 \pm 0.6^{*}$ & $0.9 \pm 0.3^{*}$ & $1.3 \pm 0.6^{*}$ \\
\hline $\begin{array}{l}\text { Vitamin } \mathrm{D}_{3} \\
(\mathrm{nmol} / \mathrm{l})\end{array}$ & $100-120$ & $95 \pm 6^{* *}$ & $99 \pm 4^{* *}$ & $104 \pm 4^{* *}$ \\
\hline
\end{tabular}

Note: $*$ and $* *$ are the confidence levels of the difference in indicators relative to the norm (p), respectively $\leq 0.001$ and $\leq 0.0$.

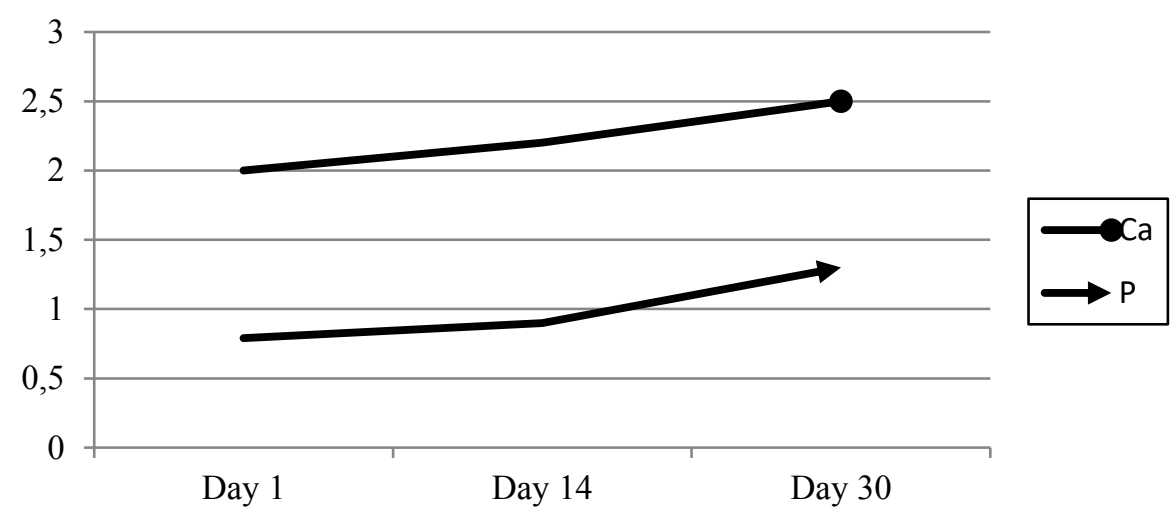

Fig. 1. Change of $\mathrm{Ca}$ and $\mathrm{P}$ level

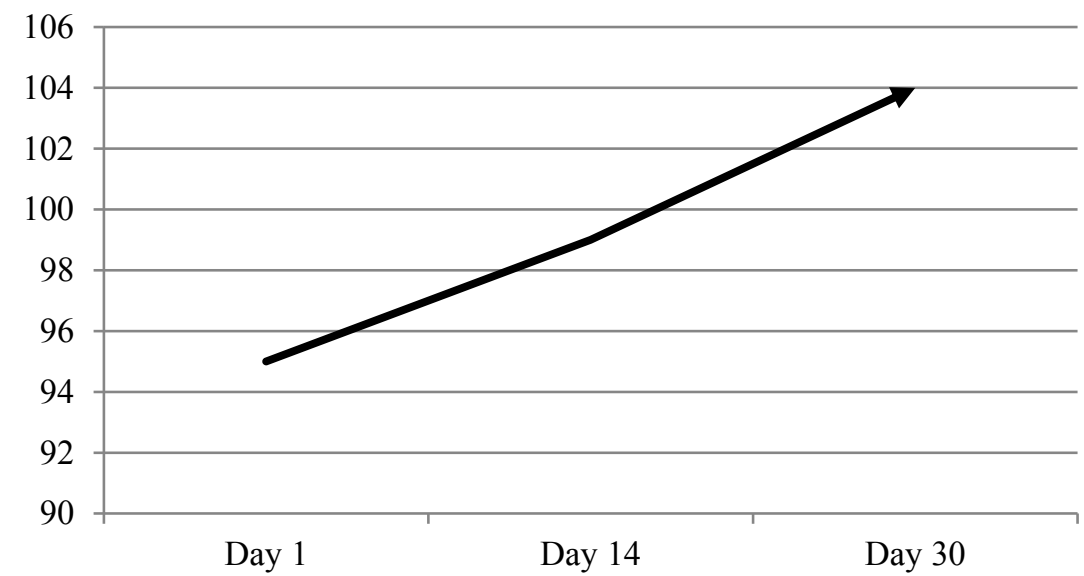

Fig. 2. Change in vitamin $D_{3}$ levels 
From Table 1 and Figures 1, 2, it can be seen that in all animals, with the treatment of phytocalcevitis, the ratio of $\mathrm{Ca}$ and $\mathrm{P}$ began to equalize, the intake of vitamin $\mathrm{D}_{3}$ in the body in the form of the drug Vitatrin improved the absorption of $\mathrm{Ca}$ and $\mathrm{P}$, which led to the formation of a strong bone callus at the site of the fracture. By day 30, the indicators of the level of $\mathrm{Ca}$ and $\mathrm{P}$ rose to the lower limits of the norm. Therefore, in the treatment of fractures of any complexity, it is necessary not only to study the X-ray image, but also to conduct a biochemical blood test for the content of the level of $\mathrm{Ca}, \mathrm{P}$ and vitamin $\mathrm{D}_{3}$ and regulate their intake into the body with vitamin and mineral supplements to form a strong bone callus.

After removing the plaster cast, all the animals had joint stiffness, soreness, and partial muscle hypotrophy. When walking, all the animals did not lean on a limb. Physical rehabilitation became necessary to restore the normal range of motion, ensure mobility, and dynamic stability of the limbs. It is widely used in clinical medicine, but is not sufficiently developed in veterinary medicine. The main principles of rehabilitation are the following: early start of rehabilitation activities, comprehensive use of all available and necessary funds, individualization of the program, phasing, integrity and continuity throughout all stages of rehabilitation. The plan of rehabilitation measures should be based on the assessment of functional disorders and the stages of the regeneration process.

We developed a rehabilitation program that included:

- physical exercises;

- massage

Physical exercises included exercises to increase the volume of movement in the injured limb, muscle strength, functional exercises, and correction of abnormal biomechanics. A necessary condition was the restoration of proprioception, when nerve impulses from joints, muscles, and tendons provide information about the position of the body in space. Nerve endings and pathways are damaged as a result of trauma and disrupt the segmental transmission of nerve impulses, which leads to a violation of balance, decreased coordination, impaired perception of joints position, changes in reflexes when performing movements. Proprioceptive exercises do not have a stressful effect on damaged tissues and help to increase the effectiveness of strength and endurance exercises. The weight load contributes to the increase of proprioceptive capabilities. Moreover, the restoration of proprioception avoids repeated injuries and secondary dysfunction.

Massage - a set of different special techniques that have a mechanical, dosed and reflex effect on various areas of the animal's body surface, produced by the masseur. Manual massage is based on the principle of using the segmental structure of the body and reflex reactions that occur when exposed to certain reflexogenic zones. It was performed using the techniques of stroking, rubbing, kneading and vibration.

To determine the effectiveness of rehabilitation procedures, 2 groups of animals were formed. The first group consisted of animals whose owners refused to perform rehabilitation procedures. The second group included dogs whose owners performed daily rehabilitation activities for their pets. Pet owners were trained to conduct rehabilitation activities at home. At the same time, the program was adapted to the needs and disorders of the animal, it is specific, progressive and accessible to owners.

In animals with a fracture of the ulnar and radial bones, the plaster cast was removed after 4 weeks. They were prescribed the following restorative procedures: light massage in the form of stroking and rubbing the phalanges of the fingers with progress to the bones of the wrist and forearm, not massaging them, but massaging from the lateral side: the radial extensor of the wrist, the general extensor of the fingers, the lateral extensor of the fingers, the ulnar extensor of the wrist; from the plantar side: the ulnar flexor of the wrist and the superficial flexor of the fingers, and from the medial side: the radial flexor of the wrist, the radial extensor of the wrist wrists, brachioradialis muscle. To massage the tip of the elbow, 
triceps and biceps with rubbing movements. Functional gymnastics consisted of light, painfree flexion and extension of the joints of phalangeal bones, wrist and elbow joints from 10 to 20 times with a daily increase in the number of repetitions.

In animals with fractures of the pelvic limb bones, the plaster cast was removed after 6 weeks and the massage was advised to begin with the phalanges of the fingers by stroking, lightly rubbing to the top and massaging from the lateral side: the long extensor of the fingers, the long flexor of the thumb; from the plantar side: the calf muscle, the tendons of the semi-tendon muscle and the biceps femoris; from the dorsal side, a light rubbing massage of the cranial tibial muscle. When massaging gluteus, sartorial muscle, musculus quadriceps femoris, semi-tendon muscle, semitendinous muscle, semimembranosus muscle, m. gracilis to stretch the limb as much as possible and make a little effort to fully knead these muscles. Massage included stroking, rubbing with increasing pressure, tapping, pinching for 3-5 minutes 2-3 times a day. Functional gymnastics consisted of light, painfree flexion and extension of the joints of the phalanges of fingers, ankle and knee joint from 5 to 20 times with a daily increase.

Owners of all animals were advised to do not only massage and functional gymnastics, but also to put the animal on three limbs, bending the healthy limb so that the animal leans on the affected limb and keep it so from 1 to 5 minutes daily, as well as increase the time of walking outside - this improves muscle tone, restores hypotrophic muscles, and also trains the cardiovascular system.

Thus, an active lifestyle, good balanced feeding, and being outdoors led to a complete recovery of all dogs. In the animals of the second group, which the owners carried out daily rehabilitation measures, restoration of limb muscles strength and increase in the volume of movements in the joints were observed 10-14 days after the removal of the plaster cast. In dogs of the first group, lameness persisted for up to 3 weeks, and in one dog, which had a closed metaphysical fracture of the fibula and a fracture of the metaphysical part of the femur with displacement, lameness was observed for up to 2 months.

\section{Conclusions}

Vitamin and mineral metabolism in animals has a significant impact on the state of the bone system and the body as a whole.

With a decrease in the level of $\mathrm{Ca}, \mathrm{P}$ and vitamin $\mathrm{D}_{3}$ in the blood, the bones become brittle and break quickly. Therefore, when diagnosing fractures and for better treatment prescription, it is necessary to conduct a biochemical blood test.

For medical treatment, it is necessary to use preparations of $\mathrm{Ca}, \mathrm{P}$ and vitamin $\mathrm{D}_{3}$ to increase their level in the blood and, accordingly, the rapid formation of a strong bone callus and bone restoration.

After removing the plaster cast, the animals had joint stiffness, soreness, and partial muscle hypotrophy. Physical rehabilitation is necessary to restore the normal range of motion, ensure mobility, and dynamic stability of the limbs.

The rehabilitation program developed by us includes physical exercises and massage. In dogs whose owners carried out daily rehabilitation measures, restoration of the strength of the limb muscles, increase in the volume of movements and complete recovery occurred 2 times faster than in animals in which rehabilitation measures were not carried out.

\section{References}

1. V.A. Lukyanovsky, A.D. Belov, I.M. Belyakov, Diseases of the bone system of animals, 254 (M., Kolos, 1984) 
2. A.D. Belov, V.A. Lushchnovsky, Bone fractures, General veterinary surgery, 472-496 (M., Agropromizdat, 1990)

3. N.V. Shamsutdinova, F.F. Valiullina, Eurasian Scientific Association, 10, 272-273 (2019)

4. K.A. Petrakov, P.T. Salenko, S.M. Paninsky, Operative surgery with topographical anatomy of animals, 2nd ed., reprint. and add., 453 (M., KolosS, 2013)

5. P.M. Taylor, J.E. Houlton, Traumatology of dogs and cats, 208 (M., Aquarium-Print, 2016)

6. P.A. Revell, Pathology of the bone ,114-140 (M.: Meditsina, 1993)

7. D.L. Millis, A. CiupercaI, Vet Clin North Am Small Anim Pract. Jan, 45(1), 1-27 (2015)

8. A.A. Vishnevsky, Injuries and fractures: treatment and recovery, 192 (St. Petersburg: Nevsky Prospekt, 2004)

9. G.G. Shalamova, E.V. Smelkova, A.A. Salieva, Scientific Notes of the KGAVM, 244, 243-247, (2020)

10. L.L. Artamonova, O.P. Panfilov, V.V. Borisova, Therapeutic and adaptive-healthimproving physical culture: textbook.manual for university students studying in the specialty "Physical culture", 389 (Moscow, Vlados-Press Publishing House, 2014) 